\title{
SUBSETS OF FIRST COUNTABLE SPACES
}

DANIEL E. WULBERT ${ }^{1}$

I. Summary. Let $X$ be a topological space. If $X$ is second countable, it is known [3, Theorem VIII, p. 246] that any sequence of subsets of $X$ contains a subsequence which is topologically convergent. In $\S I I$ of this note we show that if $X$ is first countable, any net of subsets of $X$ contains a subnet which is topologically convergent. (See §II for definitions.)

Let $Q$ denote the space of compact nonempty subsets of a Hausdorff space $X$, topologized with the finite topology. In §III we characterize the spaces $X$ for which $Q$ is first countable. It previously had been thought that the first countability of $X$ was sufficient to imply first countability of $Q$ [4, Proposition 4.5.3, p. 162]. Appropriate counter examples are found in §III.

II. Topological convergence of sets. Let $\left\{A_{j}: j\right.$ in $\left.J\right\}$ be a net of subsets (not necessarily closed or nonempty) of a topological space $X$. $\lim S\left(A_{j}\right)=\{x$ in $X$ : for every neighborhood $U$ of $x$ and any $k$ in $J$ there is a $j$ in $J$ such that $j \geqq k$ and $\left.A_{j} \cap U \neq \varnothing\right\}$,

and

$\lim I\left(A_{j}\right)=\{x$ in $X:$ for every neighborhood $U$ of $x$ there is a $k$ in $J$ such that $j \geqq k$ implies $\left.A_{j} \cap U \neq \varnothing\right\}$.

The net $\left\{A_{j}\right\}$ is termed topologically convergent if $\lim S\left(A_{j}\right)=\lim I\left(A_{j}\right)$. If $\left\{A_{j}\right\}$ is topologically convergent, the set $\lim S\left(A_{j}\right)$ is called the limit of $\left\{A_{j}\right\}$. We note that $\lim S\left(A_{j}\right)$ may be the empty set.

If $X$ is second countable, it is known that every sequence of subsets of $X$ contains a subsequence which is topologically convergent [3, Theorem VIII, p. 246]. The purpose of this section is to prove the following theorem.

THEOREM. If $X$ is first countable, every net of subsets of $X$ contains a topologically convergent subnet.

Proof. We will first show that if $\left\{B_{k}\right\}$ is any net of subsets of $X$, then there is a subnet $\left\{B_{h}: h\right.$ in $\left.H\right\}$ of $\left\{B_{k}\right\}$ such that

Received by the editors August 2, 1967.

1 This research was sponsored by the Air Force Office of Scientific Research under grant AFOSR 1109-67. The author is grateful to L'Ecole Polytechnique de l'Université de Lausanne where he was a guest during the preparation of this manuscript. 


$$
\bigcup_{g \text { in } H} \bigcap_{h \geq \rho} B_{h}=\bigcap_{\rho \text { in } H} \underset{h \geq g}{\bigcup} B_{h} .
$$

To prove the above, put

$$
\begin{aligned}
\psi_{k}(x) & =1 & & \text { if } x \text { is in } B_{k} \\
& =0 & & \text { otherwise. }
\end{aligned}
$$

Let $P=\{0,1\}^{E}$. Being a product of compact spaces, $P$ is compact. Hence there is a subnet $\left\{\psi_{h}: h\right.$ in $\left.H\right\}$ of $\left\{\psi_{k}\right\}$ which is convergent in the product topology on $P$. Let

$$
B_{h}=\left\{x \text { in } X: \psi_{h}(x)=1\right\} .
$$

By direct computation one can verify that $\left\{B_{h}: h\right.$ in $\left.H\right\}$ is a subnet of $\left\{B_{k}\right\}$ satisfying the property stated above.

Now let $\left\{A_{j}: j\right.$ in $\left.J_{0}\right\}$ be a net of subsets of $X$. For each $x$ in $X$, let $\{V(x, k): k=1,2, \cdots\}$ be a countable base for the neighborhood system for $x$. We may assume that $V(x, k)$ contains $V(x, k+1)$. Now for $j$ in $J_{0}$ and $k=1,2, \cdots$ let

$$
B(k, j, 0)=\left\{x \text { in } X: V(x, k) \cap A_{j} \neq \varnothing\right\} .
$$

From the first part of the proof there is a subnet of $\left\{B(1, j, 0): j\right.$ in $\left.J_{0}\right\}$ say $\left\{B(1, j, 1): j\right.$ in $\left.J_{1}\right\}$ such that

$$
\underset{g \text { in } J_{1}}{\bigcup} \bigcap_{h \geq g} B(1, j, 1)=\bigcap_{g \text { in } J_{1}} \underset{h \geqq g}{\bigcup} B(1, j, 1) .
$$

Having constructed the net $\left\{B(i-1, j, i-1): j\right.$ in $\left.J_{i-1}\right\}$, we consider the net of subsets $\left\{B(i, j, i-1): j\right.$ in $\left.J_{i-1}\right\}$. As above, this net admits a subnet $\left\{B(i, j, i): j\right.$ in $\left.J_{i}\right\}$ such that

$$
\underset{g \text { in } J_{i}}{\cup} \bigcap_{h \geqq g} B(i, j, i)=\bigcap_{g \text { in } J_{i}} \underset{h \geq g}{\bigcup} B(i, j, i) .
$$

Let $D=\times\left\{J_{i}: i=1,2, \cdots\right\}$ have the product ordering. For $d$ in $D$, let $d(i)$ denote the $i$ th coordinate of $d$. Let $I$ denote the positive integers, and let $I \times D$ have the product ordering. For $s=(i, d)$ in $S$, let $R(s)=(i, d(i), i)$. Let $B=\bigcap_{i=1}^{\infty} \bigcup_{g}$ in $J_{i} \bigcap_{h \geq g} B(i, h, i)$. We intend to show that the net $\left\{A_{R(s)}: s\right.$ in $\left.S\right\}$ converges topologically to $B$. Since this net is a subnet of $\left\{A_{j}: j\right.$ in $\left.J_{0}\right\}$, this will complete the proof of the theorem.

Now suppose $x$ is in $B$. We will show that every neighborhood of $x$ eventually meets $A_{R(s)}$. From the definitions of $B$ and $B(i, j, i)$ we have that, for every positive integer $k$,

$$
\left\{V(x, k) \cap A_{(k, j, k)}: j \text { in } J_{k}\right\}
$$


eventually consists of nonempty sets. Now $\left\{A_{R(i, d)}: i>k, d\right.$ in $\left.D\right\}$ is a subnet of $\left\{A_{(k, j, k)}: j\right.$ in $\left.J_{k}\right\}$. Hence $\left\{V(x, k) \cap A_{R(s)}: s\right.$ in $\left.S\right\}$ eventually consists of nonempty sets as we intended to show.

If $x$ is not in $B$, there is a $k$ such that $x$ is not in

$$
\underset{g \text { in } J_{k}}{\bigcup} \bigcap_{h \geqq g} B(k, h, k)=\bigcap_{g \text { in } J_{k}} \underset{h \geqq g}{U} B(k, h, k) .
$$

Hence $\left\{V(x, k) \cap A_{(k, j, k)}: j\right.$ in $\left.J_{k}\right\}$ eventually consists of empty sets. Since $\left\{A_{R(i, d)}: i>k, d\right.$ in $\left.D\right\}$ is a subnet of $\left\{A_{(k, j, k)}: j\right.$ in $\left.J_{k}\right\}$, we have that $\left\{V(x, k) \cap A_{R(s)}: s\right.$ in $\left.S\right\}$ eventually consists of empty sets.

We have shown that if $x$ is not in $B$, then $x$ has a neighborhood which eventually has empty intersection with $A_{R(s)}$. We conclude that $\left\{A_{R(s)}\right\}$ converges to $B$. This completes our proof.

We do not know, if under the hypothesis of the theorem, every sequence of subsets of $X$ contains a convergent subsequence.

III. Finite topology. Let $X$ be a Hausdorff space, and let $Q$ denote the collection of nonempty compact subsets of $X$. For a finite collection of open sets in $X\left\{U_{i}: i=1,2, \cdots, n\right\}$, we define

$$
\begin{aligned}
& {\left[U_{1}, U_{2}, \cdots, U_{n}\right]} \\
& \quad=\left\{K \text { in } Q: K \cap U_{i} \neq \varnothing, i=1,2, \cdots, n \text {, and } K \subseteq \bigcup_{i=1}^{n} U_{i}\right\} .
\end{aligned}
$$

The collection of sets

$$
\left\{\left[U_{1}, U_{2}, \cdots, U_{n}\right]: U_{i} \text { open in } X \text { for } i=1,2, \cdots, n\right\}
$$

is a base for a topology on $Q$ called the finite (Vietoris) topology. We henceforth assume that $Q$ has the finite topology.

It is known $[4, \S 4]$ that $Q$ is completely regular, metrizable, compact, separable, or second countable if and only if $X$ is respectively completely regular, metrizable, compact, separable, or second countable. In this section we characterize the spaces $X$ for which $Q$ is first countable.

A subset $P$ of $X$ is said to have a countable exterior base if there is a countable family of open sets in $X$ whose intersection is $P$ and such that every open set containing $P$ also contains some member of the countable family. If $X$ is compact, a closed set $P$ has a countable exterior base if and only if it is a $G_{\delta}$ set.

TheOREM. In order that $Q$ is first countable, it is necessary and sufficient that the following conditions are satisfied:

(1) Every compact subset of $X$ is separable.

(2) Every compact subset of $X$ has a countable exterior base. 
Proof. Suppose that $\left\{\left[U(i, j): i=1,2, \cdots, n_{j}\right]: j=1,2, \cdots\right\}$ is a countable base in the finite topology for the neighborhood system of an element $P$ in $Q$. Let $b(i, j)$ be a point in the intersection of $U(i, j)$ and $P$. We will show that $\left\{b(i, j): i=1,2, \cdots, n_{j} ; j=1,2, \cdots\right\}$ is dense in $P$. Let $V$ be an open set in $X$ which has nonempty intersection with $P$. Since $[X, V]$ is a neighborhood of $P$, there is a $k$ such that $\left[U(i, k): i=1,2, \cdots, n_{k}\right]$ is contained in $[X, V]$. If for each $i=1,2, \cdots, n_{k}$, there is a point $y_{i}$ in $U[i, k]-V$, then $\left\{y_{1}, y_{2}, \cdots, y_{n_{k}}\right\}$ would be a compact set contained in $\left[U(i, k): i=1,2, \cdots, n_{k}\right]$ but not in $[X, V]$. This, however, is not possible. Hence $V$ contains one of the sets $U(i, k): i=1,2, \cdots, n_{k}$, and $V$ contains one of the points $b(i, j)$. Hence $P$ is separable.

If $W$ is an open set containing $P$, then $[W]$ is a neighborhood of $P$. For some $k\left[U(i, k): i=1,2, \cdots, n_{k}\right]$ is contained in $[W]$ and $\bigcup_{i=1}^{n_{k}} U(i, k)$ is contained in $W$. It follows that $P$ has a countable exterior base.

To prove the converse let $P$ be a compact set in $X$, and assume that $X$ satisfies properties (1) and (2) in the statement of the theorem. Let $\{U(i): i=1,2, \cdots\}$ be a countable family of open sets which form a countable exterior base for $P$. Let $\{x(i): i=1,2, \cdots\}$ be a countable dense subset of $P$. For $i=1,2, \cdots$ let $\{V(i, j): j=1,2, \cdots\}$ be a countable base for the neighborhood system in $X$ of the point $x(i)$. Let $W=\bigcup\{V(i, j): i=1,2, \cdots ; j=1,2, \cdots\}$ Now

$$
\left\{\left[U(i), W_{1}, W_{2}, \cdots, W_{n}\right]: W_{1}, W_{2}, \cdots, W_{n} \text { in } W \text { and } i=1,2, \cdots\right\}
$$

is a countable base in the finite topology for the neighborhood system for $P$. This completes the proof.

In the following corollaries let $X$ be a compact Hausdorff space.

Corollary. If $Q$ is first countable then $X$ is a first countable, separable, perfectly normal space (see $[2, p .134])$.

COROLlary. If $Q$ is first countable, $Q$ is separable.

ExAmple. Let $X=[0,1] \times[0,1]$ be ordered lexicographically and given the order topology. Then $X$ is first countable, Hausdorff, and compact but not separable [2, p. 164].

ExAmple. Let $X$ be as in the example above. Let

$$
P=\{(r, i) \text { in } X: i=0 \text { or } i=1 \text { and } 0 \leqq r \leqq 1\} .
$$

Now $P$ is compact and separable, but $P$ does not have a countable exterior base. For if $V$ is an open set containing $P$ there can exist at most finitely many real values $x_{i}$ such that $0<x_{i}<1$ and there is a $y_{i}$ 
for which $0<y_{i}<1$ and $\left(x_{i}, y_{i}\right)$ is not in $V$. (If there did exist an infinite number of such points $x_{i}$ we could construct a sequence of points not in $V$ that converged to a point in $P$. This of course is not possible.) Therefore any countable intersection of open sets in $X$ which contains $P$ must also contain points not in $P$. Hence $P$ does not have a countable exterior base.

\section{REFERENCES}

1. G. Choquet, Convergences, Ann Inst. Fourier (Grenoble) 23 (1947)/(1948), $55-112$.

2. J. R. Kelley, General topology, Van Nostrand, New York, 1955.

3. C. Kuratowski, Topologie. I, 2nd ed., Monogr. Mat., Vol. 20, P. W. N., Warsaw, 1948.

4. E. Michael, Topologies on spaces of subsets, Trans. Amer. Math. Soc. 71 (1951), 151-182.

University of Texas 